\title{
Prevalence of sarcopenia in a Scottish cohort with chronic kidney disease (CKD) receiving haemodialysis and its association with clinical parameters
}

\author{
S. Smith ${ }^{1}$, P. Rayson ${ }^{1}$, J. Goddard ${ }^{2}$ and I. Davidson ${ }^{1}$ \\ ${ }^{1}$ School of Health Sciences, Queen Margaret University, Edinburgh, EH21 6UU and ${ }^{2}$ Renal Unit, Edinburgh Royal \\ Infirmary, Edinburgh, EH16 4SA, UK
}

The presence of chronic kidney disease (CKD) has been suggested to elicit early onset reductions in lean body mass leading to disease related sarcopenia ${ }^{1}$ and poor functional performance. We investigated the prevalence of sarcopenia in a Scottish cohort receiving long term ( $>6$ months) haemodialysis and its association with age, dialysis vintage, comorbidity and inflammation.

The diagnosis of sarcopenia was based on the European consensus definition and diagnosis ${ }^{2}$. Handgrip as a marker of muscle strength was measured pre dialysis. Height and weight were measured post dialysis for the calculation of body mass index (BMI). Calf circumference (CC) and mid arm muscle circumference (MAMC) were measured post dialysis as anthropometric estimates of muscle mass. Dual frequency bioelectrical impedance analysis (DFBIA) was also measured post dialysis to derive skeletal muscle index (SMI). Functional performance was assessed using the 6-minute walk test and number of sit-to-stand transitions performed in 1 minute. Comorbidity scores were determined using the Charlson co-morbidity index scoring system, monthly high sensitivity $\mathrm{C}$-reactive protein (hsCRP) results were used to determine the presence of inflammation and dialysis vintage was calculated from the first date renal replacement therapy was initiated.

Sixty-four patients were recruited 39 male \& 25 female with a mean age of 54 years $16(\mathrm{SD})$ and BMI of $27.6 \mathrm{Kg} / \mathrm{m}^{2} 6.3(\mathrm{SD}), \mathrm{mean}$ hsCRP was $11.6 \mathrm{mgL} 5 \cdot 1$ (SD), mean dialysis vintage was 75.0 months 84.8 (SD). $43.8 \%$ were identified as sarcopenic, with $26.6 \%$ presenting with severe sarcopenia. Sarcopenic obesity was present in $20 \cdot 3 \%$. Those with sarcopenia performed fewer sit-to-stand transitions and walked less distance in 6 minutes than those without sarcopenia. Whilst a significant difference in SMI was observed between those with and without sarcopenia, no difference in calf circumference or mid arm muscle circumference was evident.

\begin{tabular}{|c|c|c|c|c|c|c|c|c|c|c|c|c|}
\hline & \multicolumn{2}{|c|}{6 min walk $(\mathrm{m})$} & \multicolumn{2}{|c|}{ Sit to stand (n) } & \multicolumn{2}{|c|}{$\mathrm{CC}(\mathrm{cm})$} & \multicolumn{2}{|c|}{ Handgrip $(\mathrm{Kg})$} & \multicolumn{2}{|c|}{ SMI(units) } & \multicolumn{2}{|c|}{ MAMC $(\mathrm{cm})$} \\
\hline & Mean & SD & Mean & SD & Mean & $\mathrm{SD}$ & Mean & $\mathrm{SD}$ & Mean & $\overline{\mathrm{SD}}$ & Mean & $\mathrm{SD}$ \\
\hline Sarcopenia & 255 & $115^{*}$ & $13 \cdot 0$ & $10 \cdot 3 *$ & $34 \cdot 9$ & $5 \cdot 2$ & $22 \cdot 8^{*}$ & $8 \cdot 0$ & $7 \cdot 1^{*}$ & $1 \cdot 8$ & $31 \cdot 0$ & $6 \cdot 1$ \\
\hline No sarcopenia & 381 & 110 & $20 \cdot 7$ & $11 \cdot 5$ & $36 \cdot 1$ & $4 \cdot 1$ & $32 \cdot 6$ & $11 \cdot 6$ & 8.4 & $2 \cdot 3$ & $31 \cdot 2$ & $5 \cdot 8$ \\
\hline
\end{tabular}

* Mean values significantly different from non-sarcopenic individuals-p $<0.05$ Independent test.

Binomial logistic regression revealed the presence of sarcopenia to be associated with age $(\mathrm{p}<0 \cdot 01)$ and Charlson co-morbidity index $(\mathrm{p}<0.05)$, but not with length of time patients had been receiving dialysis (vintage) or hsCRP.

These results indicate a high prevalence of secondary disease related sarcopenia in a relatively young dialysis population. In this cohort, the presence of secondary sarcopenia was associated with age and comorbidities but not with the presence of inflammation or dialysis vintage. These results also suggest that anthropometric markers of muscle mass are unlikely to be sufficiently sensitive to diagnosis sarcopenia. The results require confirmation in a larger more diverse population, but suggest that work is required to identify targeted interventions which can halt or prevent the development of secondary disease related sarcopenia in this group.

1. Carrero JJ, Chimielewski M, Alexsson J et al. (2008). Clin Nut 27 557-564.

2. Cruz-Jentoft AJ, Bayens J-P, Bauer JM et al. (2010). Age and Ageing 39 412-423. 